\title{
On the use of ordinal scoring scales in social life cycle assessment
}

\author{
Rickard Arvidsson ${ }^{1}$ \\ Received: 20 September 2018 / Accepted: 7 November 2018 / Published online: 8 December 2018 \\ (C) The Author(s) 2018
}

\section{Introducing ordinal measurement scales}

In his widely adopted classification of measurement scales, Stevens (1946) differs between nominal, ordinal, interval and ratio scales (Table 1). An ordinal scale consists of values which order is known, but not the distance between the values. Examples of ordinal scales include (never, seldom, sometimes, often, always) and (1, 2, 3, 4, 5), where the latter is an example of when integers are used. Although the $(1,2,3,4,5)$ example might seem like a scale where the distance between the scores is known, this is not necessarily the case. For example, if that scale was used for ranking the experience of staying at a hotel, there is no guarantee that the respondents have interpreted 2 as exactly twice as nice a stay as 1 . From a mathematical point of view, using ordinal values for calculations such as addition, subtraction, multiplication and division does not give meaningful results in a strict sense since the distance between two values on an ordinal scale is unknown. Simply put, since the distance between $X$ and $Y$ on an ordinal scale is unknown, neither $X+Y, X-Y, X \times Y$ nor $X \quad Y$ can be meaningfully calculated.

Despite this mathematical limitation, ordinal scales are frequently used for addition, subtraction, multiplication and division in various research fields. One example is the use of Likert scales in psychology, where often 5-item scales are used to assess attitudes, for example in psychometric measurements of risk perception. Another example is in happiness research, which is largely based on peoples' ordinal scoring of their subjective wellbeing. The motivation behind the use of ordinal scales in these fields is generally that there is an underlying, latent variable, such as peoples' "true" happiness, which is difficult or impossible to measure directly (Fayers

Responsible editor: Mary Ann Curran

Rickard Arvidsson

rickard.arvidsson@chalmers.se

1 Environmental Systems Analysis, Chalmers University of Technology, Vera Sandbergs Allé 8, 41296 Gothenburg, Sweden and Hand 2002). Instead, scorings on an ordinal scale are used as surrogates for the latent variable. Stevens (1946) himself writes that such an "illegal" use of ordinal scales can be given "pragmatic sanction" since it can sometimes lead to "fruitful results."

However, considering the unknown distance between ordinal values and resulting mathematical limitation note above, the use of ordinal scales in these fields is not uncontroversial. To quote Jamieson (2004) from her paper about the (ab)use of Likert scales: "I remain convinced ... that the average of 'fair' and 'good' is not 'fair-and-a-half'; this is true even when one assigns integers to represent 'fair' and 'good'." Kuzon et al. (1996) even consider the use of parametric analysis (e.g. calculating mean and standard deviation) for ordinal data one of the "seven deadly sins of statistical analysis." For comprehensive descriptions of the "ordinal controversy," see Knapp (1990) and Kampen and Swyngedouw (2000).

\section{Ordinal scales in current social LCA}

In environmental life cycle assessment (LCA), environmental impacts are typically quantified using ratio scales, which are the most commonly encountered scales in the natural sciences and engineering. In social LCA, the use of ordinal scoring scales has instead become the dominate practice. Ordinal scales employed in social LCA include, but are not limited to, (0, 1, 3, 5, 7, 9) (Hosseinijou et al. 2014), (low, medium, high, very high) (Martínez-Blanco et al. 2014), (1, 2, 3, 4) (Ramirez et al. 2014) and (1, 2, 3, 4, 5, 6) (Ciroth and Franze 2011). Often, ordinal scale values are depicted as integers or translated into integers at some point in the assessment (e.g. very negative effect $\rightarrow 6$ ). The values of the ordinal scales are furthermore often highlighted using traffic light colours and the assignment of values is typically based on whether certain underlying socially related criteria are fulfilled.

An example of the use of ordinal scales in social LCA can be found in the Handbook for Product Social Impact Assessment (Fontes 2016). There, the ordinal scale $(+2,+1$, 
Table 1 Four types of measurement scales. Modified from Stevens (1946). Note that each main feature is also held by subsequent scales

\begin{tabular}{ll}
\hline Scale & Main features \\
\hline Nominal & Naming, enabling evaluation of membership $(=$ or $\neq)$ \\
Ordinal & Rank order, enabling comparison $(>$ or $<)$ \\
Interval & Equally sized intervals, enabling addition and subtraction \\
Ratio & A non-arbitrary zero, enabling multiplication and division
\end{tabular}

$0,-1,-2)$ is used to score so-called social topics in each life cycle phase based on qualitative criteria for company behaviours. The scores are then typically aggregated into social topic scores for the whole life cycle, which can be further weighted into aggregated stakeholder group scores. The different stakeholder group scores can then be weighted and added into a total score. Another example is the Social Hotspots Database (SHDB), in which values from the ordinal scale (low risk, medium risk, high risk, very high risk) are assigned to so-called social issues for each life cycle phase (Benoit Norris and Norris 2015). This assignment is based on the values of underlying parameters, which are often in the form of quantitative ratio-scale data. These ordinal values are then converted into respective ordinal integer values $(0.1$, $1,5,10)$. The social issues can be aggregated over the entire life cycle by multiplying with the number of working hours required in each life cycle phase and summing up. Moreover, the social issues can then be weighted and summed into a total Social Hotspots Index. Similar procedures can be found in other social LCA approaches, often involving summing of scores and/or multiplication by weighting/scaling factors for different social topics/issues/stakeholders.

\section{Recommendations for the future}

Considering the complexity of social phenomena and the difficulty of capturing many of them using ratio scales, it is possible that the use of ordinal scales in social LCA cannot be avoided completely. However, their current use in social LCA involves addition, subtraction, multiplication and division of ordinal values, which is controversial due to the unknown distance between such values. It is therefore recommended that this use is limited to situations where the latent variable cannot be meaningfully captured by ratio-scale indicators. For a number of social topics, there are already readyto-use ratio-scale indicators available. Three examples are provided below, which relate to three suggested social topics in the guidelines on social LCA: Local employment, health and safety, and equal opportunities/discrimination (Benoît et al. 2009). Local employment in social LCA can be assessed by calculating the distribution of labour hours along product life cycles, which is a ratio-scale indicator with time as unit
(Hunkeler 2006). The share of labour hours conducted locally (given some geographical reference point) can then be calculated. Health impacts can be assessed using the ratio-scale indicator disability-adjusted life years (DALY), quantifying years of life lost and disability in the unit time, which has been applied in a number of social LCA case studies (Norris 2006; Arvidsson et al. 2018). Regarding equal opportunities and discrimination, Kruse et al. (2008) proposed a number of cost-related ratio-scale indicators measured in terms of monetary value. One of them was gendered labour costs, which calculates life cycle labour costs broken out by males and females, revealing the share of labour costs benefitting females. It thus captures parts of the gender aspect of equal opportunities. In addition to already-existing ratio-scale indicators in social LCA, it is recommended that further research is put into developing new ones that capture important social topics.

Acknowledgements The author is grateful to Anna Furberg, Sverker Molander and an anonymous reviewer for valuable comments on the text.

Open Access This article is distributed under the terms of the Creative Commons Attribution 4.0 International License (http:// creativecommons.org/licenses/by/4.0/), which permits unrestricted use, distribution, and reproduction in any medium, provided you give appropriate credit to the original author(s) and the source, provide a link to the Creative Commons license, and indicate if changes were made.

Publisher's Note Springer Nature remains neutral with regard to jurisdictional claims in published maps and institutional affiliations.

\section{References}

Arvidsson R, Hildenbrand J, Baumann H, Islam KMN, Parsmo R (2018) A method for human health impact assessment in social LCA: lessons from three case studies. Int J Life Cycle Assess 23(3):690-699

Benoît C, Mazijn B, Andrews ES, Barthel L-P, Beck T, Ciroth A, Cucuzzella C, Gensch C-O, Hébert J, Lesage P, Manhart A, Mazeau P, Methot A-L, Moberg A, Norris G, Parent J, Prakash S, Reveret J-P, Spillemaeckers S, CML U, Valdivia S, Weidema BP (2009) Guidelines for social life cycle assessment of products. United Nations Environment Programme and Society of Environmental Toxicology and Chemistry, Nairobi

Benoît Norris C, Norris GA (2015) Chapter 8: The social hotspot database. In: Murray J, McBrain D, Wiedmann T (eds) The sustainability practitioner's guide to social analysis and assessment, 1 st edn. Common Ground, Champaign

Ciroth A, Franze J (2011) LCA of an ecolabeleded notebook - consideration of social and environmental impacts along the entire life cycle. GreenDeltaTC GmbH, Berlin

Fayers PM, Hand DJ (2002) Causal variables, indicator variables and measurement scales: an example from quality of life. J Royal Stat Soc: Series A 165(2):233-253

Fontes J (2016) Handbook for product social impact assessment Version 3.0. PRé Sustainability, Amersfoort

Hosseinijou S, Mansour S, Shirazi M (2014) Social life cycle assessment for material selection: a case study of building materials. Int J Life Cycle Assess 19(3):620-645 
Hunkeler D (2006) Societal LCA methodology and case study. Int J Life Cycle Assess 11(6):371-382

Jamieson S (2004) Likert scales: how to (ab)use them. Med Educ 38: $1217-1218$

Kampen J, Swyngedouw M (2000) The ordinal controversy revisited. Qual Quant 3487-102

Knapp TR (1990) Treating ordinal scales as interval scales: an attempt to resolve the controversy. Nurs Res 39:39121-39123

Kruse SA, Flysjö A, Kasperczyk N, Scholz AJ (2008) Socioeconomic indicators as a complement to life cycle assessment - an application to salmon production systems. Int J Life Cycle Assess 14(1):8-18

Kuzon WMJ, Urbanchek M, McCabe S (1996) The seven deadly sins of statistical analysis. Ann Plast Surg 37:265-272
Martínez-Blanco J, Lehmann A, Muñoz P, Antón A, Traverso M, Rieradevall J, Finkbeiner M (2014) Application challenges for the social Life Cycle Assessment of fertilizers within life cycle sustainability assessment. J Clean Prod 69(0):34-48

Norris G (2006) Social impacts in product life cycles - towards life cycle attribute assessment. Int J Life Cycle Assess 11(1):97-104

Ramirez PKS, Petti L, Haberland NT, Ugaya CML (2014) Subcategory assessment method for social life cycle assessment. Part 1: methodological framework. Int J Life Cycle Assess 19(8):1515-1523

Stevens SS (1946) On the theory of scales of measurement. Science 103(2684):677-680 